\title{
TENDÊNCIA DE CRESCIMENTO DE FILHOS DE MÃES ADOLESCENTES
}

\section{GROWTH TREND OF INFANTS OF ADOLESCENTS MOTHERS}

\author{
Paulo Rogério Gallo ${ }^{1}$ \\ Claudio Leone ${ }^{1}$ \\ Hugo Amigo ${ }^{2}$
}

Gallo PR, Leone, C, Amigo, H. Tendência de crescimento de filhos de mães adolescentes. Rev Bras Crescimento Desenvolv Hum. 2009; 19(2):297-305.

\section{Resumo:}

Objetivo: comparar o crescimento de lactentes filhos de mães adolescentes com o de filhos de mães adultas nos primeiros dois anos de vida. Métodos: estudo de coorte histórica realizado entre 1998 e 2000, comparando o crescimento de dois grupos de crianças do nascimento até completar dois anos de idade. Um dos grupos era constituído por filhos de mães adolescentes (n:127) e o outro por filhos de mães adultas (n:181). Ambos os grupos eram acompanhados regularmente nas duas Unidades Básicas de Saúde da Universidade de São Paulo, integradas ao sistema de público de saúde. Os dados de peso e comprimento, coletados sistematicamente, foram analisados sob a forma de escore Z (NCHS/OMS, 1978). Equações (curvas) de regressão descrevendo o crescimento individual de cada criança foram definidas a partir dos dados coletados. Posteriormente, os valores de escore $\mathrm{Z}$ de peso e comprimento em idades exatas (mensais) foram estimados por interpolação, utilizando-se as equações individuais obtidas. Resultados: ao nascimento e aos 24 meses os dois grupos não apresentaram diferença nas médias dos escores $\mathrm{Z}$ de peso e comprimento que, contudo, eram inferiores às do referencial $(\mathrm{p}<0,001)$. Neste período, apresentaram desempenhos diferentes de crescimento, com uma tendência pior para os filhos de adolescentes, que se afastava da mediana do referencial de maneira significante, $(p=0,0008)$. Conclusão: Os lactentes de mães adolescentes apresentaram um pior desempenho de crescimento até os dois anos de vida, quando comparados com o dos filhos de mães adultas.

Palavras-chave: mães; adolescente; crianças com deficiências; estatura-idade.

Departamento de Saúde Materno-Infantil , Faculdade de Saúde Pública, Universidade de São Paulo. Departamento de Saúde Materno-Infantil , Faculdade de Saúde Pública, Universidade de São Paulo.

Departamento de Nutrição. Faculdade de Medicina. Universidade do Chile, Santiago.

Titulo resumido: Tendência de crescimento em lactentes;

Financiamento FAPESP (Fundação de Amparo à Pesquisa do Estado de São Paulo) no. 98/05500-0

Correspondência para: Paulo Rogério Gallo, Departamento de Saúde Materno-Infantil , Faculdade de Saúde Pública, Universidade de São Paulo. Av. Dr. Arnaldo, 715. CEP: 01246-904. São Paulo, SP, Brasil. Tel.: 0113081 2451. FAX: 0113085 0240. E-mail: prgallo@usp.br 


\begin{abstract}
:
Objetive: to compare the growth of children of adolescent mothers with that of children of adult mothers in the first two years of life. Methods: A historical cohort study, carried out between 1998 and 2000, comparing the growth of 2 groups of children from birth to two years. One group is constituted by children of adolescent mothers $(n=127)$ and the other, by children of adult mothers $(n=181)$. Both groups were regularly monitored in two Basic Health Units of the University of São Paulo, which attend a typical target group that seeks assistance in public health services. The weight and length data were analyzed in $\mathrm{Z}$ scores (NCHS/WHO). Growth curves for each child were modeled based on regression equations from birth up to two years of age. Results: At birth and at 24 months of age, the groups were anthropometrically similar, but their measures were below the NCHS referential data $(\mathrm{p}<0.001)$. However, throughout the two years the two groups showed a different growth rhythm, with the growth deficit presented by the adolescents' children being manifested by a tendency for distancing from the referential data $(\mathrm{p}=0.0008)$. Conclusion: The children of adolescent mothers have a worse growth performance compared with that of the children of adult mothers in the first two years of life.
\end{abstract}

Key words: mother; adolescent; disabled children; stature by age.

\section{INTRODUÇÃO:}

Filhos de mães adolescentes vêm adquirindo particular relevância no âmbito da assistência à saúde, tanto em decorrência da antecipação do ciclo reprodutivo entre as mulheres, que vem acarretando gestações em adolescentes cada vez mais jovens, quanto pelos problemas psicossociais, econômicos e biológicos que derivam desta situação., ${ }^{1,2}$ Esta relevância se torna ainda maior quando se considera o progressivo aumento nas taxas de fertilidade que se observa nesta faixa etária, que também ocorre no Brasil. ${ }^{3}$

Diversos estudos indicam que os filhos de mães adolescentes apresentam maiores riscos biológicos, tais como de prematuridade, de baixo peso ao nascer, de problemas respiratórios neonatais, entre outros ${ }^{4,5}$. Além disto, estes riscos podem se agravar ainda mais, em decorrência dos problemas sociais que as adolescentes enfrentam durante a gravidez. Problemas de ordem psicossocial podem persistir também após o nascimento, mesmo que sejam, parcial e/ou temporariamente, compensados pela intensidade das relações sociais de solidariedade da comunidade, na organização do apoio destinado à adolescente e ao seu filho. ${ }^{4}$

Todos estes fatores adversos, particularmente os pós natais, podem afetar o desempenho de crescimento das crianças filhas de mães adolescentes e, portanto, podem resultar numa restrição de seu potencial de crescimento, que se manifestaria já nos primeiros anos de vida.

Apesar do processo de transição nutricional, restrição do crescimento, como um todo, mas particularmente estatural, ainda tem elevada prevalência em regiões da América Latina. Diversos estudos têm indicado que é comum esta condição se instalar logo no início da vida das crianças destas regiões e que sua recuperação se torna difícil após os cinco ou seis anos de idade. ${ }^{6,7,8}$

Assim, o objetivo é descrever e comparar até os dois anos de idade, um grupo de filhos de mães adolescentes com um de filhos de mães adultas. 


\section{MÉTODO:}

Trata-se da análise por conglomerado, dos dados de uma coorte histórica com pareamento, a partir de um estudo realizado entre 1998 e 2000, com coleta retrospectiva de dados, comparando o crescimento desde o nascimento até os 24 meses de idade de dois grupos de crianças. Um grupo constituído por filhos de mães adolescentes, por ocasião do nascimento da criança (mulheres com menos de vinte anos de idade) com outro, de filhos de mães adultas também por ocasião do nascimento da criança (mulheres entre 20 e 35 anos de idade). Os dados de ambos os grupos de crianças foram retirados de 3988 prontuários clínicos de crianças que tiveram acompanhamento segundo o protocolo assistencial (REF) para lactentes, nos dois Centros de Saúde Escola (CSE), da Universidade de São Paulo, na cidade de São Paulo, Brasil. Todas as mães eram moradoras de uma mesma área urbana.

Critérios de inclusão e exclusão: Em ambos os grupos foram incluídas apenas crianças nascidas a termo, adequadas para a idade gestacional, que tivessem mantido assistência regular, segundo protocolo assistencial nos dois primeiros anos de vida e que tivessem sido anotadas em seus prontuários pelos profissionais de saúde dos CSE, pelo menos sete avaliações de peso e comprimento entre o nascimento e os 24 meses de idade e com avaliações registradas com intervalos máximo de três meses entre as medições.

Foram excluídas crianças declaradas adotadas, as com enfermidades orgânicas crônicas, metabólicas ou genéticas, as nascidas prematuras e/ou com peso de nascimento inferior a 2500 gramas.

O grupo que compôs a coorte de filhos de mães adolescentes foi constituído por 127 crianças. Este número correspondia a cerca de $20 \%$ do total de filhos de mães adolescentes matriculados nas duas Unidades de Saúde durante o período de estudo.
O grupo controle de crianças filhas de mães adultas foi selecionado num segundo momento, a partir da identificação dos filhos das adolescentes, utilizando como critérios de pareamento sexo, data de nascimento (+/- 2 meses), ordem de nascimento, bairro de residência, local de atendimento e idade de início do acompanhamento pós-natal (aceitou-se +/2 meses como limites de variabilidade).

Ao final, o grupo de filhos de mães adultas foi constituído por 181 crianças. Este número correspondia a $6,3 \%$ do total de crianças filhas de mães adultas matriculadas nas duas Unidades, durante o período de estudo.

Os Centros de Saúde Escola selecionados da Universidade de São Paulo prestam assistência primária de maneira regular à população urbana de sua área de abrangência. Por serem Unidades de capacitação de alunos de graduação (nutrição, enfermagem e medicina) e de pós-graduação lato e stricto senso, há prioridade no atendimento de crianças de famílias de baixo nível sócio-econômico, cuja renda familiar mensal per capita não ultrapassa U\$ 80,00 , com a maioria de seus componentes apresentando nível de escolaridade inferior ao fundamental. Segundo dados dos serviços pouco mais de 1/3 dos atendimentos são de famílias residentes em áreas de favela próximas das Unidades .

As medições de peso e comprimento, bem como os respectivos registros, são regularmente efetuadas pelos profissionais da Saúde das Unidades, que por sua vez, dada a natureza dos serviços, são treinados para obedecer e ensinar técnicas reconhecidas internacionalmente para a tomada de medidas antropométricas $^{9}$. Habitualmente as medidas das crianças são tomadas sob a supervisão de estudantes e estagiários dos setores.

Amostragem: O tamanho mínimo de amostra estimado foi de 125 crianças para cada grupo, mães adolescentes e mães adultas, de maneira a garantir um poder de teste (1-b) de 0,8 e um nível de significância (a) de 0,05, para 
discriminar uma diferença de pelo menos $1 / 3$ de desvio padrão, para ambos os parâmetros (peso e comprimento), entre os dois grupos.

Para descrever a curva de crescimento de peso e de estatura de cada criança, foram definidas individualmente, equações de regressão a partir dos dados coletados, recalculando-se a seguir, a partir de equações individualizadas, por interpolação, os valores estimados para as idades exatas (mês a mês) de 0 a 24 meses. Posteriormente, foram feitas comparações a partir do escore $\mathrm{Z}$ de comprimento e peso, para a idade e sexo, calculado a partir dos valores do referencial antropométrico do National Center for Health Statistics (NCHS) de 1978, proposto pela Organização Mundial da Saúde (OMS). A tendência de evolução do escore $Z$ do crescimento linear foi estabelecida automaticamente, ajustando-se os dados para expressar a melhor regressão linear que expressasse o conjunto dos resultados para cada idade exata.

As médias de escore $\mathrm{Z}$ de comprimento e peso, entre os grupos de filhos de mães adolescentes e de mães adultas, nas idades exatas de $0,6,12$, 18 e 24 meses, foram comparadas pelo teste t de Student e por ANOVA.

Para o processamento dos dados foram utilizados os programas Excel ${ }^{\circledR}$, CurveExpert ${ }^{\circledR}$ 1.3 e SPSS $^{\circledR} 9.0$.

Referencial utilizado: Para análise dos resultados foi utilizado referencial recomendado pelo National Center for Health Statistics
(NCHS) a partir da publicação de 1977. A motivação da escolha foi definida em função desse referencial ter sido o mais utilizado em publicações da época em que os dados foram colhidos. Esta decisão, na análise dos dados desse artigo, possibilita que os resultados sejam analisados à luz de outras publicações do final da década de 1990 e primeiros anos da década de 2000. Uma segunda observação sobre o uso desse referencial pode ser remetida ao fato de que o objetivo principal deste artigo ser o de comparar, entre si, o perfil de crescimento de dois grupos de crianças.

\section{RESULTADOS:}

A tabela 1 mostra a comparação de algumas das principais características das crianças e suas mães. Como se observa, a única diferença estatisticamente significante, já esperada, foi a média de idade dos grupos de mães por ocasião do nascimento de seus filhos.

As amostras apresentaram proporções similares de meninos e meninas e nenhuma diferença significante quanto ao nível médio de escolaridade materna.

A análise das condições de nascimento no tocante ao peso e ao comprimento dos filhos de adolescentes e das mães adultas mostram que a situação não difere entre os dois grupos ( $(\mathrm{=}=0,999)$. Entretanto, observa-se que ambos os grupos são significativamente de

Tabela 1: Características gerais das amostras de crianças

\begin{tabular}{lccc}
\hline Variáveis & Mães adolescentes & Mães adultas & Significância \\
\hline idade das mães(anos) & $18.0(1,5)^{*}$ & $26.0(4,1)^{*}$ & $\mathrm{P}<0.001$ \\
Proporção de meninos & $50.4 \%$ & $48.1 \%$ & $\mathrm{~ns}$ \\
Anos de escolaridade materna6.7 $(2.6)^{*}$ & $6.0(3.1)^{*}$ & $\mathrm{~ns}$ \\
Anos de escolaridade Paterna6.6 $(2.9)^{*}$ & $6.0(3.1)^{*}$ & $\mathrm{~ns}$ \\
\hline * média (desvio padrão) & & \\
ns (não significante). & &
\end{tabular}


menor comprimento ao nascer quando comparados com o referencial internacional $(\mathrm{p}<0,001)$ do NCHS-78 (Tabela 2).

Aos 24 meses, a situação é relativamente similar, os dois grupos mantêm as diferen- ças significativas com o padrão internacional, mas, não diferem entre si (tabela 3). Apesar disto, ao longo dos 24 meses, verificou-se que as crianças de mães adolescentes e as de mães adultas apresentaram diferentes desempenhos

Tabela 2. Média e Desvio Padrão em escores Z, do peso e do comprimento ao nascer dos filhos de adolescentes e de adultas e do referencial NCHS.

\begin{tabular}{|c|c|c|c|}
\hline & $\begin{array}{c}\text { Mães adolescentes (a) } \\
\text { Média (sd) }\end{array}$ & $\begin{array}{l}\text { Mães adultas (b) } \dagger \\
\text { Média (sd) }\end{array}$ & $\begin{array}{l}\text { Referencial (c) } \dagger \\
\text { Média (sd) }\end{array}$ \\
\hline Peso & $-0.02(0.760)^{*}$ & $-0.05(0.820)^{*}$ & $0(1.0)^{*}$ \\
\hline Comprimento† & $-0.54(0.714)^{* *}$ & $-0.63(0.796)^{* *}$ & $0(1.0)$ \\
\hline ns; & & & \\
\hline ** $\quad \mathrm{ns}$ & & & \\
\hline$\dagger \quad$ a ou b ve & $\mathrm{p}<0.001$ (comprimento) & & \\
\hline
\end{tabular}

Tabela 3: Média e Desvio Padrão em escores Z do peso e do comprimento aos 24 meses, segundo referencial do NCHS dos filhos de adolescentes e de adultas.

\begin{tabular}{lccc}
\hline & $\begin{array}{c}\text { Mães adolescentes } \\
\text { Media (sd) }\end{array}$ & $\begin{array}{c}\text { Mães adultas } \\
\text { Media (sd) }\end{array}$ & $\begin{array}{c}\text { Referencial } \\
\text { Media (sd) }\end{array}$ \\
\hline Peso & $-0.03(1.106)^{*}$ & $0.02(1,155)^{*}$ & $0(1.0)^{*}$ \\
Comprimento & $-0.38(0.940)^{* *}$ & $-0.29(1.077)^{* *}$ & $0(1.0)$ \\
\hline
\end{tabular}

* ns

** ns

de crescimento, com estas últimas atingindo um escore $\mathrm{Z}$ de comprimento maior que a sua média de escore $Z$ ao nascimento $(p=0,0008)$; enquanto que a média de escore $Z$ de comprimento de filhos de adolescentes não apresentou diferenças estatísticas entre nascimento e 24 meses (figura 1 ).

O crescimento de peso e comprimento não revelou diferenças significativas intragrupos de filhos de mães adolescentes quando comparadas as duas faixas de idade materna (menores de 20 anos ou 20 anos ou mais). As médias de escores $Z$ de peso destes grupos aos 24 meses de idade, foram res- pectivamente de 0,05 e -0,11 e as de comprimento: -0,44 e -0,42.

A figura 1 descreve a evolução da média de escore $Z$ de comprimento de 0 a 24 meses para ambos os grupos, mostrando um período de crescimento compensatório nos primeiros dois meses de vida o qual, no entanto, não foi suficiente para atingir a mediana de crescimento esperada de acordo com o referencial. Ao atingir os quatro meses de vida, as crianças das mães adultas evidenciaram tendência a manter o canal de crescimento que haviam alcançado, enquanto que o crescimento das crianças de mães adolescentes manteve 


\section{Figura 1: Evolução do escore $Z$ de estatura para idade de filhos de adolescentes e de adultas entre 0 e 24 meses,} São Paulo, 1999-00

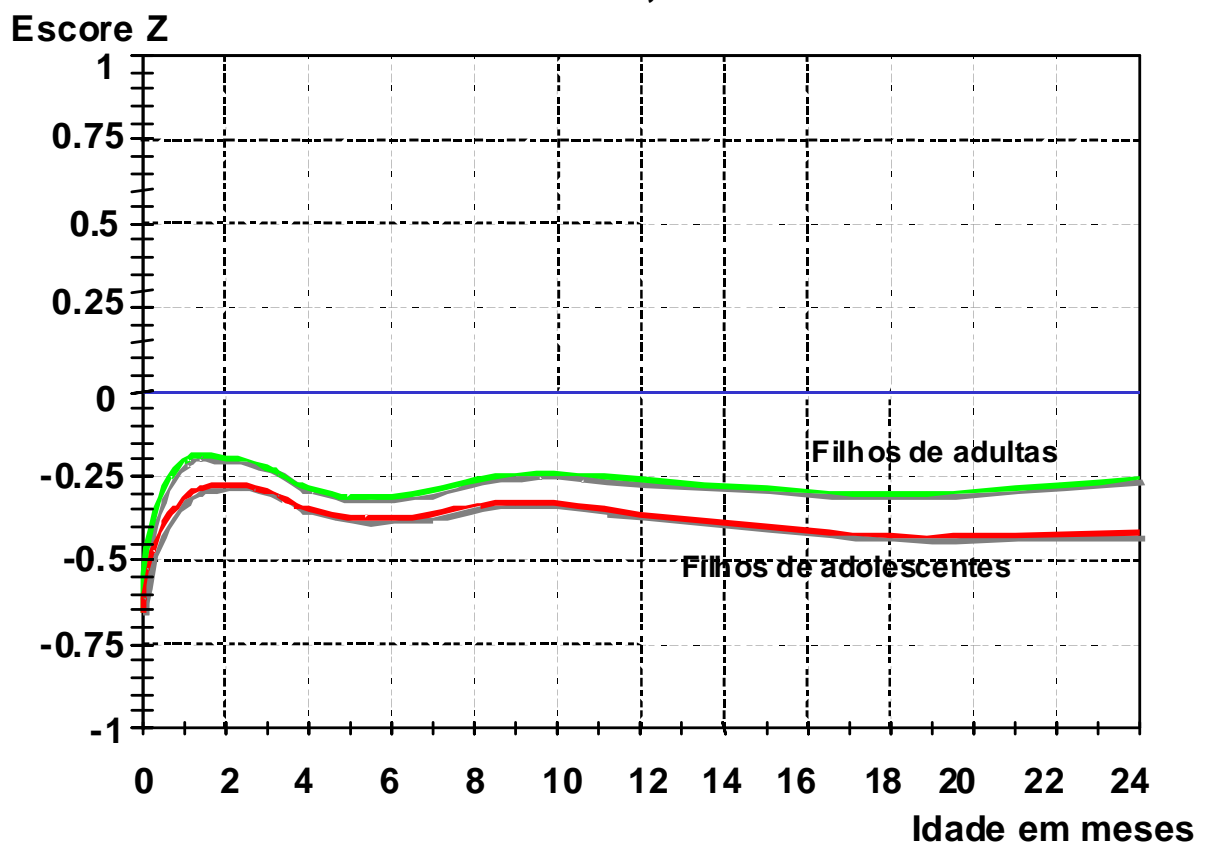

uma tendência decrescente de crescimento. Observa-se, assim, uma tendência divergente de crescimento entre os grupos a partir do segundo semestre de vida.
A tendência de evolução do escore $Z$, estabelecida por regressão linear, Figura 2, confirma estas diferenças no crescimento entre os dois grupos. A tendência da regressão

Figura 2: Tendência de crescimento do comprimento para a mães adoléscentes e de adultas. São Paulo, 1999-00.

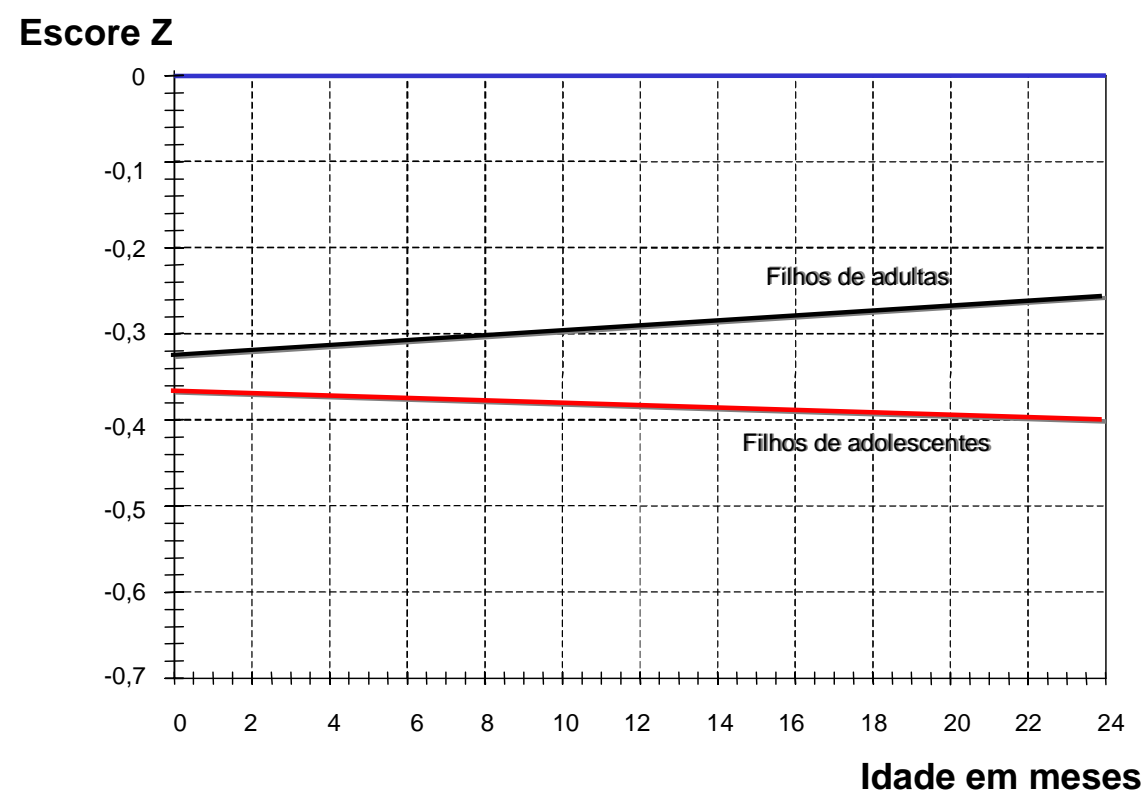


linear de crescimento das crianças de mães adultas não difere estatisticamente do referencial $(F=0,222 ; p=0,64)$, enquanto que a das crianças de mães adolescentes apresenta diferença significante $(F=6,658 ; p=0,01)$.

\section{DISCUSSÃO:}

O primeiro aspecto a ser considerado é que, neste estudo, foram excluídos os recémnascidos pré-termo e de baixo peso, pois se objetivava descrever e avaliar o desempenho de crescimento nos primeiros dois anos de vida de crianças filhas de mães adolescentes que potencialmente poderiam crescer adequadamente.

Quanto ao comprimento ao nascer, observou-se uma relativa desproporção com o peso. Este resultado é explicado, pelo menos em parte, pelo fato dos critérios de inclusão e exclusão terem sido estabelecidos apenas para o peso ao nascer.

O comprimento ao nascer dos dois grupos foi significativamente menor do que a mediana do referencial. Esta diferença do referencial de - 0,65 escore $Z$ pode ser decorrência de dificuldades na mensuração das crianças ao nascimento e/ou de agravos ocorridos em períodos específicos da gestação, tais como: insuficiente ganho de peso materno, tabagismo, baixa qualidade de pré-natal ou por intercorrências nutricionais. ${ }^{9,10}$

Apesar destas possibilidades, considerando que o menor comprimento ao nascer foi observado em crianças com condições relativamente satisfatórias de nascimento, independentemente do grupo de mães considerado, não pode ser descartado o papel do referencial utilizado (NCHS/OMS 1978) nos resultados dos escores $\mathrm{Z}$ de comprimento observados. As características do estudo, em cujos dados o referencial se baseou, podem gerar distorções na comparação com os valores antropométricos ${ }^{11}$ observados, particularmente no que diz respeito ao período neonatal.
Por volta dos nove meses de idade, as diferenças no potencial de crescimento dos dois grupos começam a se manifestar, quando as curvas de crescimento principiam a evidenciar tendências discrepantes. É importante considerar que a desaceleração no crescimento linear, observada aos nove meses, é decorrência de agravos iniciados alguns meses antes.

Apesar da explicação deste fenômeno fugir ao âmbito deste estudo, o fato é que possivelmente alguma situação "sui generis" afeta de maneira desigual crianças filhas de mães adolescentes e de mães adultas. Isto dificilmente poderia ser atribuído a um menor potencial genético de crescimento ou às condições de gestação e nascimento da criança, condições estas controladas no desenho do presente estudo. A hipótese mais aceitável é que existam diferenças nas circunstâncias que se referem aos cuidados diários dispensados a estas crianças ${ }^{12}$, incluindo as consequências associadas a características da transição alimentar que ocorre durante o processo de desmame ${ }^{13}$.

A magnitude da diminuição da velocidade de crescimento do comprimento fica bem evidente quando se considera o crescimento compensatório que estas crianças apresentaram até os três ou quatro meses de vida e que é perdido a partir de então. O resultado final é que o ganho total em centímetros durante o período de estudo como um todo é, em média, praticamente o esperado pelo referencial, sem que se recupere aos dois anos, principalmente nos filhos das adolescentes, a restrição de crescimento que estava presente ao nascer.

Esta falta de recuperação do déficit de crescimento pode ser atribuída às mesmas condições socioeconômicas que são as responsáveis pela baixa estatura encontrada em muitas áreas de países subdesenvolvidos ${ }^{14}$. No caso das adolescentes deste estudo, é pouco provável que esta seja a única ou a principal explicação para a restrição de crescimento de suas crianças até os dois anos. Na verdade, é possível que também as características psicológicas 
da adolescente, face ao papel que na sociedade moderna se espera da mulher, também possam ser um fator de risco adicional para o processo de crescimento de seus filhos.

A tendência de progressivo afastamento que a média dos parâmetros de crescimento dos filhos das mães adolescentes apresenta não só em relação ao referencial, mas, principalmente, também da média de crescimento observada para as crianças filhas de mães adultas provenientes de uma mesma região e de uma mesma condição social, se vier a ser constatada também em outros estudos, sugere que é necessária uma assistência especial, diferenciada, no acompanhamento de rotina de filhos de mães adolescentes. Esta atenção diferenciada se torna ainda mais importante quando se consideram as evidências da literatura que apontam para a possibilidade de ocorrer uma irreversibilidade da restrição de crescimento que se instala nesta fase da vida. , $^{8,14,15}$

Nestas análises é preciso levar em conta que as tendências de crescimento analisadas são estimadas a partir de dados coletados como parte da rotina assistencial prestada a estas crianças e, portanto sujeitas a um maior risco de erro de medida. Entretanto, este risco fica atenuado quando se considera que as Unidades em que foram coletadas são unidades de ensino, capacitadas a treinar profissionais de saúde especificamente na realização destas rotinas, executadas quase sempre de maneira padronizada, com instrumentos adequados e sob a supervisão de um profissional capacitado.

Outro aspecto importante a considerar é que o crescimento de cada criança foi estimado individualmente, com base em seus próprios dados de crescimento, buscando definir a equação da curva de regressão que melhor se ajustava aos mesmos, desta maneira minimizando os possíveis erros que medidas isoladas, discrepantes da tendência geral de crescimento da criança no período, poderiam produzir.

Finalmente vale lembrar que não se trata de uma amostra representativa do universo de filhos de mães adolescentes, mas, sim, de uma coorte proveniente de duas unidades de cuidados primários de sede universitária, portanto seus resultados devem ser interpretados com alguma cautela.

Entretanto, o fato de se tratar de recém nascidos a termo, saudáveis, acompanhados regularmente em serviços de boa qualidade assistencial e que, mesmo assim, no caso de mães adolescentes, em média apresentam restrição de seu crescimento nos dois primeiros anos de vida, sugere fortemente que se trata de uma população de risco de crescimento que provavelmente seria beneficiada por um programa diferenciado (específico) de monitoração e de cuidados de saúde durante os primeiros anos de vida.

Assim, houve evidências de crescimento diferente, indicando um crescimento menos satisfatório quando se compara o grupo de filhos de mães adolescentes com o de filhos de mães adultas, qual seja, mães em idade reprodutiva.

O estudo não permite detalhar fatores de risco que estariam envolvidos no pior desempenho do grupo dos filhos de adolescentes. No entanto, permite verificar que o pior desempenho é uma tendência que se estende de modo consistente, ao longo dos dois primeiros anos de vida das crianças. Este fato aponta que os fatores de risco estão imbricados no modo de vida dessas mães adolescentes, repercutindo negativamente nas condições de vida de seus filhos.

\section{AGRADECIMENTOS}

Os autores destinam um particular agradecimento a Vera Lúcia Machado e Flávia Muramatsu por sua importante participação na coleta e processamento dos dados do estudo.

Esta pesquisa foi realizada com o suporte da FAPESP (Fundação de Amparo à Pesquisa do Estado de São Paulo), processo número 98/05500-0. 


\section{REFERÊNCIAS:}

1. Maranhão AGK, Joaquim MMC, Siu C. Mortalidade perinatal e neonatal no Brasil. Tema 17: 6-17, 1999.

2. Martorell R, Khan LK, Schroeder DG.Reversibility of stunting: epidemiological findings in children from developing countries. Eur J Clin Nutr1994 Feb; 48 Suppl 1:S45-57

3. Sweeney PJ. Gravidez na adolescência . In: Sanfilippo JS. et al. Ginecologia pediátrica e da adolescente. Rio de Janeiro, Guanabatra Koogan, 1994. P240-7.

4. Luz AMH. Mulher adolescente : sexualidade gravidez e maternidade. EDIPUCRS. Rio Grande do Sul, 1999. $1^{\mathrm{a}}$ ed, 234p.

5. Uauy R, Albala C, Kain J. Obesity trends in Latin América: transiting from under- to overweight. J. Nutrition, 2001;131:893S-899S.

6. Center For Desease Control: Premarital sexual experience among adolescent women.United States. 1970-1988. M.M.W.R., 39:929-32,1991.

7. Eveleth PB, Tanner JM. Worldwide variation in human growth. $2^{\mathrm{a}}$ ed. Cambridge, Cambridge University Press, 1990.

8. Galletta MA, Zugaib M. Assistência à gestante adolescente. Estudos Avançados, 13(35, supl. especial): 35-6, 1999 ( taxas de fecundidade)
9. Gallo PR, Reis AOA, Leone C.

Características de seguimento pré-natal, parto e recém-nascido, de adolescentes grávidas, Município de Joinville, Estado de Santa Catarina, Brasil, 1995. Pediatria (São Paulo), vol 22 (2), p. 123-9.

10. Jelliffe, D.B. Evaluación del estado de nutricion de la comunidad. In: Organizacion Mundial de la Salud, Série de monografias n.53. Ginebra, 1968.

11. Amigo H, Bustos $\mathrm{P}$, Leone $\mathrm{C}$, Radrigan ME. Growth deficits in chilean school children. J. Nutrition 2001; 131: 251254.

12. Oliveira MLS, Bastos ACS. Práticas de atenção à saúde no contexto familiar: um estudo comparativo de casos. Psicol. Reflex. Crit. [online]. 2000, vol.13, n.1, pp. 97-107.

13. Araujo MFM, Rea MF, Pinheiro KA, Schmitz BAS. Avanços na norma brasileira de comercialização de alimentos para idade infantil. Rev. Saúde Pública [online]. 2006, vol.40, n.3, pp. 513-520.

14. Ribas DLB, Sganzerla A, Zorzatto JR, Philippi ST. Nutrição e saúde infantil em uma comunidade indígena Teréna, Mato Grosso do Sul, Brasil. Cad. Saúde Pública [online]. 2001, vol.17, n.2 , pp. 323-331.

15. Laurentino GEC, Arruda IKG, Arruda BKG de. Nanismo nutricional em escolares no Brasil. Rev. Bras. Saude Mater. Infant.. 2003, vol.3, n.4, pp. 377-385.

Recebido em: 16 de dezembro de 2008. Modificado em: 22 de março de 2009. Aceito em: 25 de maio de 2009. 